\title{
Política de qualidade e eficácia dos cuidados de saúde rural
}

\author{
Endossado pela Wonca,
}

Novembro de 2003

WONCA Working Party on Rural Practice

A Política de Qualidade e Eficácia dos Cuidados de Saúde Rural foi desenvolvida pelo Grupo de Trabalho de Medicina Rural.

O objetivo é fornecer uma estrutura que estimule o desenvolvimento de metas diretas e indiretas e de avaliaçōes da qualidade e efetividade da prática da medicina rural.

Em nome do Grupo de Trabalho de Medicina Rural da Wonca, esperamos que este documento estimule as ideias e ajude no esforço para o sucesso da proposta da Organização Mundial de Saúde de "Saúde para todos os povos" e da Declaração de Durban "Saúde para todas as populações rurais em 2020".

\section{Sumário executivo}

Este documento é uma contribuição para o debate sobre a qualidade e a efetividade dos cuidados de saúde em todo o mundo. Tendo como referencial o objetivo da OMS de saúde para todos os povos, o GT de Medicina Rural da WONCA concorda que alcançar um cuidado de saúde rural de alta qualidade e efetividade é um enorme desafio para os países desenvolvidos e em desenvolvimento. Saúde rural, definida de maneira ampla, inclui o bem-estar físico, mental e social, mas também está relacionada ao grau de conectividade/interatividade que o indivíduo tem com a família, os amigos, o trabalho e a comunidade.

Moradores de áreas rurais de todo o mundo em geral possuem menos acesso aos cuidados de saúde e piores estado de saúde e desfechos em saúde quando comparados com as populaçôes urbanas. Avaliaçôes da qualidade e da efetividade do cuidado de saúde rural não estão disponíveis, nem são dirigidos ou aplicados à realidade rural. O documento sugere parâmetros para avaliaçôes diretas e indiretas dos cuidados de saúde em áreas rurais que possam estar em concordância com a meta de "Saúde para todas as populaçóes rurais em todo o mundo". Este intento inclui o contexto da ruralidade, o estado de saúde em áreas rurais, os desfechos de saúde nessas circunstâncias, os serviços de saúde, a força de trabalho, a educação dos trabalhadores, a tecnologia de informação e a infraestrutura no âmbito da ruralidade, pesquisa, financiamento e organização dos cuidados de saúde e a satisfação do consumidor desses cuidados.

Uma série de vinhetas de saúde de todo o mundo é apresentada, a fim de ressaltar os problemas de saúde a partir de uma perspectiva mundial.

\footnotetext{
$\overline{\text { Como citar: Working Party on Rural Practice WONCA. Política de qualidade e eficácia dos cuidados }}$

de saúde rural. Rev Bras Med Fam Comunidade. 2013;8(Suppl 1):15-24.

Disponível em: http://dx.doi.org/10.5712/rbmfc8(1)728
} 


\section{Introdução}

A Organização Mundial de Médicos de Família ${ }^{1}$ e a Organização Mundial de Saúde ${ }^{2}$ propuseram metas ambiciosas para a saúde de todas as populações rurais no século 21. Essas metas são mais difíceis de serem atingidas naqueles locais em que a maior parte da população vive em áreas rurais. A melhoria da saúde das populaçóes rurais implica na promoção do bem-estar físico, mental e social, de acordo com a definição ampliada de saúde da OMS. A qualidade da saúde das populaçóes rurais está fundamentada em determinantes muito amplos, incluindo não apenas a ausência de doença, mas a 'conectividade/ interatividade', entendida como o relacionamento do indivíduo com sua família, amigos, trabalho e comunidade. Populações rurais saudáveis são produto de uma geração de pais que propiciem aos seus filhos um relacionamento fundamentado em bases sólidas e prazerosas, em que eles vivam, brinquem e trabalhem de forma produtiva ao longo de suas vidas. Melhorar a saúde das populaçôes rurais requer atividade cooperativa multidimensional de todas as áreas da nossa sociedade, necessitando atençấo para os aspectos ambientais, econômicos, dos recursos disponíveis, educacionais e cuidados de saúde.

Populaçóes com necessidades diferenciadas devem ter alta prioridade, em especial as necessidades de saúde de povos indígenas, minorias e comunidades rurais isoladas, visando atingir o mesmo estado de saúde desejado para o resto da população. Prover um cuidado de saúde efetivo e de alta qualidade é um desafio gigantesco seja em países desenvolvidos ou em desenvolvimento. As comunidades rurais, quando comparadas às comunidades urbanas, possuem menos acesso aos cuidados de saúde e, por isso, piores estado de saúde e desfechos de saúde. Avaliaçóes da qualidade e da efetividade dos cuidados de saúde não estão bem estudadas, dirigidas e nem são aplicadas ao contexto rural. Esta Política oferece um desenho para o desenvolvimento e a implementação de iniciativas de melhoria da qualidade da saúde rural. Ela descreve e apresenta critérios diretos e indiretos de saúde rural que estáo alinhadas com a proposta da WONCA de "Saúde para todos os povos" e a Declaração de Durban de "Saúde para toda a População Rural até 2020" (Tabela 1). A qualidade e a efetividade do cuidado à saúde rural podem ser medidas diretamente usando o estado de saúde rural e os desfechos em saúde rural. Medidas indiretas, incluindo a disponibilidade de serviços de saúde, de mão de obra, organização, infraestrutura, pesquisa e financiamento, também são importantes. Ao final, alguns casos clínicos são apresentados para destacar o contexto da saúde rural e ilustrar o desafio de atingir a qualidade e a efetividade do cuidado à saúde rural quer nos países desenvolvidos ou em desenvolvimento.

\begin{tabular}{|c|c|}
\hline & Parâmetros Diretos e Indiretos de Cuidados de Saúde Rural \\
\hline 1. & Contexto Rural \\
\hline 2. & Estado de saúde rural \\
\hline 3. & Desfecho em cuidados de saúde rural \\
\hline 4. & Serviços em cuidados de saúde rural \\
\hline 5. & Recursos Humanos em Saúde rural \\
\hline 6. & Formação de Recursos Humanos em Cuidados de saúde rural \\
\hline 7. & Infraestrutura e tecnologias de informação em cuidados de saúde rural \\
\hline 8. & Pesquisa em cuidados de saúde rural \\
\hline 9. & Financiamento de saúde rural \\
\hline 10. & Organização de cuidados em saúde rural \\
\hline 11. & Satisfação do consumidor da saúde rural \\
\hline
\end{tabular}

\section{Parâmetros Diretos e Indiretos de Cuidados de Saúde Rural}

\section{Contexto rural}

Objetivo: Compreensão do contexto rural, incluindo as diferenças entre rural e urbano e entre diferenças específicas entre diferentes ruralidades, assim como melhorar o modelo e a oferta dos serviços de saúde, e, em última análise, dos desfechos de saúde nas áreas rurais. 
Parâmetros: Geografia e demografia das populaçóes rurais, incluindo distribuiçáo por sexo e idade; sociologia e psicologia, incluindo atitudes e valores; nível de educação; indicadores socioeconômicos de riqueza; taxas de emprego/ desemprego; profissão; qualidade da água, moradia, saneamento e existência de rede elétrica.

Comentários: Fatores socioeconômicos são determinantes fundamentais da saúde. Áreas rurais geralmente possuem taxas menores que as nacionais de escolaridade e renda per capita e taxas mais altas do que as nacionais de desemprego, além de proporcionalmente mais ocupaçôes de baixa renda. As populaçóes rurais também possuem frequentemente risco de acidentes, lesôes e traumas relacionados a atividades como a pesca, agricultura, mineração, silvicultura e, ainda, acidentes automobilísticos e desastres ambientais. Existem ótimos exemplos de vilas e áreas rurais onde são encontrados, excepcionalmente, desfechos favoráveis, e essas áreas precisam ser estudadas para a compreensão e a duplicação das causas de seus sucessos.

A definição do que é rural varia enormemente de país para país, o que gera dificuldade fazer comparações. No Canadá, por exemplo, se define atualmente, como população rural, os habitantes de áreas censitárias que não sejam regiōes metropolitanas e nem regiôes de aglomeração populacional. Essa definição engloba, essencialmente, as pequenas comunidades com menos de 10.000 habitantes que não estão localizadas nos grandes centros urbanos. Definição semelhante é usada em outros países.

\section{Estado de saúde}

Meta: "Saúde para todas as populaçôes rurais".

Parâmetros: Avaliação dos desfechos em saúde rural inclui tabelas de expectativa de vida e taxas de mortalidade, inclusive mortalidade infantil e perinatal, mortalidade materna e taxas de suicídio; incidência e prevalência de doenças, incluindo as doenças infecciosas como a tuberculose, HIV/AIDS, doenças crônico-degenerativas, como o diabete e as doenças cardiovasculares, comparando os níveis de incapacidade entre populaçóes rurais e urbanas. Avaliar estes desfechos em populaçôes indígenas (aborígines /nativos) de forma específica é importante em vários países.

Comentários: Os dados de morbimortalidade são os que mensuram de forma mais objetiva as patologias rurais e podem ser comparados com dados nacionais e internacionais de forma a destacar problemas de saúde típicos das populaçôes rurais. É necessário coletar informaçôes que permitam comparar dados rural/ urbano e rural/ rural. Em todo o mundo, populaçóes indígenas, grupos de minorias e populaçóes isoladas privadas de seus direitos possuem, geralmente, estado de saúde pior do que os de populações urbanas ou outras populaçôes rurais, o que representa um desafio particular/especial para os cuidados de saúde rural.

\section{Desfechos em cuidados de saúde rural}

Objetivo:

Parâmetros:

Comentários:
Comparar desfechos em problemas clínicos semelhantes.

São exemplos de avaliação de desfechos a morbimortalidade por doenças como o diabete, as doenças cardiovasculares e o trauma.

Quais os desfechos atingidos pelo tratamento de doenças iguais quando comparadas populaçóes rurais, populaçóes urbanas e populaçóes nacionais? Por exemplo, um morador rural que sofreu um infarto agudo do miocárdio apresenta como desfecho um estado de saúde semelhante ao quadro de outro paciente, este morador de uma área urbana? Nascituros prematuros têm o mesmo acesso a cuidados intensivos? Estes parâmetros podem ser usados para reconhecer condiçóes específicas em que os cuidados de saúde precisam ser melhorados com a finalidade de trazer a saúde rural a valores semelhantes aos obtidos em níveis nacionais e internacionais. Auditorias realizadas nos gráficos de prática/clínica rural podem ser uma ferramenta útil para a educação médica continuada e melhoria da qualidade. 


\section{Serviços Rurais de Cuidados de Saúde}

Objetivo: $\quad$ Equidade em acesso e utilização dos serviços de saúde disponíveis.

Parâmetros: Visitas de saúde e visitas de médicos de saúde em domicílio; visitas de médicos especialistas; cuidados pré-natais; taxas de procedimentos cirúrgicos; taxas de hospitalização; atividades de prevenção (como as imunizaçôes, exames preventivos cervicais e mamografias); procedimentos ambulatoriais e utilização das taxas de incidência das condiçôes sensíveis aos cuidados primários; acesso e taxas de utilização de exames de alta complexidade (como a tomografia computadorizada, a ressonância magnética e a testagem genética); uso de recursos de telessaúde e informática aplicada à saúde.

Comentários: $\quad \mathrm{O}$ uso dos serviços de saúde é um critério importante sobre o acesso que a população tem aos cuidados de saúde. Em populações iguais - mesma distribuição de gênero e idade e mesmas incidências de doença - é de se esperar uma utilização também semelhante dos serviços de saúde, supondo-se que estes sejam distribuídos de forma suficiente e acessível. Por exemplo, a disponibilidade e a utilização de cuidados primários de saúde e dos préstimos de um médico de família seriam amplamente uniformes. A grandeza com que elas diferem refletem as barreiras oriundas do acesso desigual a esses serviços.

A interpretação das diferentes taxas de procedimentos específicos e de hospitalizaçóes pode ser bastante interessante. Por exemplo, a taxa de apendicectomias deveria ser semelhante entre duas populaçóes semelhantes, com dietas também semelhantes, uma vez que as incidências seriam as mesmas e a cirurgia é não eletiva e não prorrogável. De outro lado, a taxa de cirurgias de catarata ou de prótese de quadril é muito dependente do acesso a serviços de saúde terciários. Taxas de hospitalização maiores em doenças sensíveis aos cuidados da atenção primária, como complicaçôes de diabete e asma, demonstram uma falta de serviços de cuidados primários e secundários que terminam em elevação das taxas de complicaçôes. Interpretações desses indicadores vão se tornando mais complexas na medida em que a carga populacional de doenças difere de maneira significativa.

\section{Força de trabalho em Saúde Rural}

Objetivo:

Distribuição justa da Força de Trabalho em Saúde para atender a Cuidados de Saúde

Parâmetros: Relação médico/população; taxas Médico de Família $\left(\mathrm{GP}^{*}\right) /$ população; taxa de alguns especialistas/população; taxas enfermeira/população; apoios de saúde, por exemplo, relações fisioterapeuta/população.

Comentários: A distribuição da força de trabalho básica de saúde, incluindo médicos de família/generalistas * e enfermeiros, é uma medida importante, pois todas as populaçóes necessitam de cuidados de saúde primários e preventivos. Funcionamento eficaz de equipes multidisciplinares de saúde rural é essencial. Há um papel importante para o médico de família/GP que conhece o seu/sua paciente, é capaz de gerenciar a maioria dos seus problemas de saúde e coordenar investigaçáo especializada e cuidados especializados. A dificuldade de acesso a cuidados especializados e serviços especializados da população rural é uma importante barreira à distribuição equitativa dos serviços de saúde. É claro que uma distribuição uniforme de especialistas é impraticável, como muitos serviços de especialidades de tratamento de condiçóes de baixa prevalência que só produzem uma carga de trabalho eficiente em grandes populaçóes (neurocirurgia, por exemplo) e, portanto, precisam ser centralizados para sua eficiência. Especialistas regionais, no entanto, desempenham um papel importante em muitas áreas rurais. Acesso a clínicas especializadas pode ser uma parte importante da distribuição da força de trabalho de saúde rural, bem como prover aos prestadores de cuidados de saúde rural uma importante conexão com consultores para dúvidas e educação médica continuada. Além disso, alguma regionalização e ruralização podem ser feitas por serviços de relativamente alta tecnologia especializada. Cirurgia de catarata, por exemplo, tem sido exitosamente descentralizada em alguns países, utilizando clínicas regionais e móveis. Os aumentos nos serviços de telecomunicaçóes em todo o mundo podem fazer referência e consulta à distância de um clique do mouse. Cuidadores alternativos e medicina tradicional tribal e herbal têm seu papel em muitas partes do mundo e precisam ser estudados cientificamente para a qualidade e eficácia ${ }^{1}$.

\footnotetext{
* GP significa General Pratictioner, que é o sinônimo de Médico de Família no National Health System (NHS) inglês.

** Generalistas aqui se refere ao termo GP (General Pratictioner), sinônimo de Médico de Família no NHS inglês.
} 


\section{Pessoal de Saúde Rural - Educação e Formação}

Objetivo: $\quad$ Educação e formação de um número suficiente de médicos rurais devidamente qualificados e outros membros da força de trabalho rural de saúde.

Medidas: $\quad$ Recrutamento de pessoas do meio rural para a medicina e outras ocupaçóes de saúde, de acordo com a porcentagem rural da populaçáo; curriculum de medicina rural nos serviços de treinamento prestados na graduação; pós-graduação em medicina rural com um fluxo de prática definido; oferta de treinamento complementar em anestesiologia, gineco-obstetrícia, cirurgia, etc para o médico rural; enfermeira rural e profissionais de apoio à saúde, de acordo com a população rural.

Comentários: Médicos rurais ofertam maior gama de serviços e carregam maior nível de responsabilidade clínica, atuando em relativo isolamento profissional, quando comparados com seus congêneres metropolitanos. Prática em áreas rurais, onde especialistas e serviços especializados são limitados ou distantes, requer educação médica específica orientada para o meio rural para produzir um número suficiente de médicos e outros profissionais de saúde com formação adequada. Recrutamento de indivíduos de origem rural e educação médica rural elevaram a escolha da prática rural como uma carreira e foram eficazes na educação de mais médicos e outros profissionais de saúde com os conhecimentos, habilidades e interesse para continuar a prática rural como uma carreira ${ }^{3}$.

\section{Infraestrutura de Saúde Rural e Tecnologia da Informação}

Objetivo: Clínicas/instalaçóes/pessoal e apoio de infraestrutura de rede para fornecer cuidados de saúde rurais eficazes e manter condiçôes atraentes e sustentáveis de trabalho para os profissionais de saúde rural.

Medidas: Instalaçóes clínicas, pessoal de apoio clínico e estrutura; instalaçôes hospitalares regionais; comunicação e rede de transportes para fornecer acesso a serviços especializados para pacientes criticamente doentes e gravemente feridos; e acesso e suporte da tecnologia da informação.

Comentários: A organização da infraestrutura de saúde rural, tecnologias de informaçãa e apoio são importantes contribuintes para a saúde rural. Sem o apoio da infraestrutura de alta qualidade é impossível para os profissionais de saúde rurais prestarem cuidados de saúde rurais eficazes. Além disso, a falta de infraestrutura é uma barreira significativa para recrutamento e retençáo da força de trabalho de saúde rural ${ }^{4}$.

A tecnologia da informação tem o potencial de aumentar significativamente a força de trabalho de saúde rural. Conhecimento, acesso à informaçáo e telessaúde podem melhorar os serviços e educaçáo do paciente, onde consulta direta não é possível. A telessaúde, no entanto, não é uma opção à prestação de serviços diretos primários ou consultivos ao paciente 5 .

\section{Pesquisa em Saúde Rural}

Objetivo: $\quad$ Projetos de pesquisa efetivos locais e amplos em Saúde Rural.

Medidas: $\quad$ Envolvimento de médicos locais e dos outros trabalhadores de saúde tanto na concepção quanto participação em grandes projetos locais e de saúde rural de pesquisa, com o envolvimento apropriado da comunidade.

Comentários: As populaçôes rurais têm padróes específicos de doenças, lesôes e incapacidades. Os sistemas de saúde rurais precisam de pesquisa e desenvolvimento. Os povos indígenas têm muitas vezes determinantes muito específicos de saúde e padrões de doença usuais. Cuidados de saúde eficazes são baseados em pesquisas de campo para as necessidades da populaçáo e desempenho de melhores práticas de cuidados de saúde para esse contexto. Um componente importante desta pesquisa é a promoção da pesquisa comunitária por médicos e outros profissionais de saúde rural. 


\section{Financiamento de Saúde Rural}

Objetivo: $\quad$ Financiamento equitativo aos serviços de saúde em todas as regióes.

Medidas: $\quad$ O financiamento da Saúde Rural baseado em necessidades. Equidade pode ser avaliada pela despesa per capita, baseada em necessidades comparativas documentadas.

Comentários: O financiamento da Saúde Rural precisa não apenas ter em conta a utilização per capita dos serviços de saúde, mas também infraestrutura mais ampla e apoio necessário, por causa da distância envolvida nos cuidados de saúde rural. A eficácia para a qual o financiamento de cuidados de saúde rural é distribuído é um dos principais determinantes do tamanho da força de trabalho rural de cuidados de saúde e da infraestrutura de apoio. Isto contribuirá significativamente para a quantidade e a qualidade dos cuidados de saúde rural que podem ser desempenhados e têm um grande impacto sobre a saúde da população rural.

\section{Organização da Atenção de Saúde Rural}

Objetivo: Desenvolvimento e gestáo eficaz de recursos da saúde para atender às necessidades da populaçáo rural.

Medidas: $\quad$ Deve ser definido localmente, baseado nas necessidades locais, bem como em modelos nacionais e internacionais.

Comentários: A organização global de cuidados de saúde afeta diretamente o número e a distribuição de médicos e outros profissionais de saúde e o acesso a serviços médicos, bem como os desfechos. A organização local e regional dos cuidados de saúde afeta diretamente a qualidade e a eficácia dos cuidados de saúde rural. Participação da comunidade local na posse e gestão de serviços de saúde rural é a chave para a sustentabilidade dos serviços de saúde rural. Fortes políticas e estruturas organizacionais que abordam especificamente as necessidades de cuidados de saúde rural são, portanto, necessárias em níveis local, regional e nacional 5 .

\section{Satisfação do Consumidor Rural de Saúde}

Objetivo: $\quad$ Senso de boa saúde e cuidados de saúde de qualidade acessíveis.

Medidas: $\quad$ Medidas quantitativas e qualitativas pessoais de bem-estar/acesso a/qualidade dos cuidados de saúde recebidos.

Comentários: Muito trabalho resta a ser feito, com base nas dimensóes que os pacientes acham importante. Consumidores de cuidados de saúde rurais precisam ser envolvidos em todos os aspectos de planejamento e prestação de cuidados de saúde rural. Medidas de sua satisfação (ou falta) pode ser uma poderosa ferramenta na identificação de melhorias necessárias.

\section{Futuro}

"Saúde para Todos os Povos Rurais" exige cuidados de saúde rural de alta qualidade e eficazes. Esta continua a ser uma meta desafiadora, que exige uma abordagem social total à educação, emprego e conexâo dentro da comunidade. Isso exigirá liderança local, regional, nacional e internacional. De todas as formas, na maioria dos países do mundo um grande esforço e organização são necessários para trazer a qualidade e a eficácia dos cuidados de saúde rurais comparáveis aos padrôes urbanos. Medidas da qualidade e eficácia dos cuidados de saúde rural incluem o contexto rural, o estado de saúde rural, os desfechos de saúde rural, serviços de cuidados de saúde rural, força de trabalho em saúde rural, educação da força de trabalho rural, infraestrutura e tecnologia da informação, pesquisa em saúde rural, financiamento da saúde rural e organização da atenção à saúde rural. O Grupo de Trabalho Wonca sobre Medicina Rural incentiva fortemente o uso de metas e medidas de qualidade e efetividade dos cuidados de saúde rural no desenvolvimento e avaliaçáo de programas de saúde rural. 


\section{Referências}

1. World Organization of Family Doctors - Wonca. Health for all Rural People: The Durban Declaration; 2nd World Rural Health Congress, World Organization of Family Doctors, Policy Statement; 1997, Durban. Singapore: World Organization of Family Doctors, College of Family Physicians, College of Medicine Building; 1997. Disponível em: http://www.wonca.org/working_groups/rural_training/durban_declaration.htm

2. World Health Organization - WHO. Health-for-all policy for the twenty-first century; Fifty-First World Health Assembly; 1998, World Health Organization. Geneva: The World Health Organization, Headquarters Office in Geneva (HQ); 1998. Agenda item 19. Disponível em: http://policy.who.int

3. World Organization of Family Doctors - Wonca. Training for Rural General Practice, World Organization of Family Doctors, Policy on Training for Rural Practice. Singapore: World Organization of Family Doctors, College of Family Physicians Singapore, College of Medicine Building; 1995. Disponível em: http://www.wonca.org/working_groups/rural_training/training/WONCAP.htm

4. World Organization of Family Doctors - Wonca. Rural Practice and Rural Health, Policy on Rural Practice and Rural Health. Singapore: World Organization of Family Doctors, College of Family Physicians Singapore, College of Medicine Building; 2000. Disponível em: http://www.wonca.org/ working_groups/rural_training/practice/Practi.htm

5. World Organization of Family Doctors - Wonca. Using Information Technology to improve Rural Health Care. Singapore: World Organization of Family Doctors, College of Family Physicians Singapore, College of Medicine Building, Policy Brief; 1998. Disponível em: http://www.ruralnet.ab.ca/write/ itpolicy/itpoli.htm

6. World Organization of Family Doctors - Wonca. The Health of Indigenous Peoples: The Kuching Statement; 3rd World Rural Health Congress; 1999 , Kuching. Singapore: World Organization of Family Doctors, College of Family Physicians Singapore, College of Medicine Building; 1999. Disponível em: http://www.wonca.org/working_groups/rural_training/kuching_statement.htm

\section{Vinhetas Clínicas}

\section{Canadá}

JA, um idoso de 75 anos previamente saudável chegou à emergência do hospital rural com um quadro de IAM extenso instalado. Apesar do diagnóstico e início do tratamento terem sido feitos rapidamente, sua situação continuou a se deteriorar e ele necessitou de intubação orotraqueal, que foi bem-sucedida. O médico assistente (residente em cirurgia cardíaca de plantâo naquele fim de semana substituía os médicos rurais locais) e a família acreditavam que a chance de sobrevivência de JA dependia da sua transferência para um centro de referência terciário distante dali cem quilômetros. Desconsiderando as condiçóes climáticas (uma tempestade de inverno), médico e enfermeira seguiram em uma ambulância que terminou se acidentando. O paciente morreu e o médico, a enfermeira e a equipe da ambulância sofreram traumas, incluindo traumatismo craniano e fratura de fêmur que os afastaram do trabalho por vários meses.

Comentário: Os riscos associados a uma emergência médica e a transferência para um serviço maior não costumam ser avaliados de forma adequada.

\section{Nepal}

SH, uma nepalesa de 48 anos, apresentou dor na regiáo torácica direita, na regiáo da mama. Sendo mulher e sentindo dor em mama, ela viajou para ser consultada por um famoso ginecologista, que a examinou e, de imediato, a encaminhou a um cardiologista, o que ela fez imediatamente. Este, após examinar e realizar alguns exames, prescreveu anti-inflamatório não hormonal e a tranquilizou sobre o caráter benigno da dor. Esta, porém, continuou e foi solicitada a visita de um médico clínico geral/médico de família/médico rural que colheu sua história e de imediato fez o diagnóstico de litíase biliar, que foi confirmada pela realização de um exame de ultrassonografia feita por ele. Depois de tratada de forma adequada, a paciente se viu livre da dor e, mais adiante, foi submetida ao tratamento cirúrgico da doença.

Comentário: Este caso deixa claro a importância do clínico geral/médico de família/médico rural em qualquer cenário, particularmente em um país em desenvolvimento como o Nepal. 


\section{África do Sul}

Uma mãe deu à luz um bebê prematuro em uma área urbana. $\mathrm{O}$ neonato vai ter acesso à Unidade Intensiva Neonatal e tem uma grande chance de, se necessitar de um ventilador, ter acesso a ele. $\mathrm{O}$ mesmo bebê, se nascido em uma regiáo rural, terá poucas chances de ter acesso a um centro terciário, exceto se sua mãe possuir um plano de saúde privado. Não possuindo, a única maneira de conseguir esse cuidado é ter sido a parturiente enviada a um serviço de referência ANTES do parto. Como a possibilidade de que um parto resulte em prematuro não pode ser predita, iniciado o trabalho de parto, a distância até aquele serviço e as condições de transportá-la podem fazer desse deslocamento um risco.

\section{África do Sul}

O quadro clínico de malária grave é comum em algumas áreas rurais da África do Sul. Os médicos rurais dessas regiôes têm experiência no manejo desses casos, e os médicos de zona urbana não possuem. Estes últimos, no entanto, têm acesso às UTIs. Um paciente com o risco de complicaçôes, em especial insuficiência renal, precisa ser transferido, mas os hospitais de referência sempre têm receio em recebê-lo sem estar plenamente convencido do risco. Uma vez instalada a insuficiência renal, essa transferência se torna tecnicamente mais difícil e perigosa.

\section{Nigéria}

MG, 43 anos, G4P3 ${ }^{* * *}$, todos vivos, último filho nascido há três anos, perdeu o marido há dois anos e meio. Ela está sendo acompanhada pelo clínico geral/médico de família/médico rural com a queixa de que a perda do esposo ainda interfere em sua vontade de conseguir um novo parceiro. Ela, que engravidou de seu namorado e foi pedida em casamento, ainda não havia iniciado o pré-natal devido à sua certeza de que o falecido marido cumpriria a promessa de causar algum mal a ela e a seu novo bebê. Ela acredita, firmemente, que somente a religião poderá ajudá-la.

$\mathrm{Na}$ igreja que frequenta, é acompanhada por uma cuidadora que lhe prescreveu uma dieta e uma série de oraçóes e afirma que ela deve dar à luz na igreja, pois somente assim estará livre do risco de ver o medo que tem ser concretizado.

Ao fim da gravidez e no momento do parto, ela já estava há 48 horas com contraçóes irregulares e perda de líquido e a criança tinha uma apresentação de face com a qual sua cuidadora não soube lidar, mas não queria ficar, ou deixar sua Igreja, em situação constrangedora. A paciente solicitou, então, que o médico viesse até a Igreja e ali realizasse seu parto, pedido que ele atendeu.

Comentário: Respeitar as crenças de nossos pacientes pode salvar vidas.

\section{Egito}

SM, um fazendeiro de 63 anos, chegou a um lugarejo situado a vinte e cinco quilômetros da cidade mais próxima e foi internado no hospital local com um quadro clínico iniciado há dois dias de febre alta, prostração, dores de cabeça, fortes dores no corpo, dor abdominal e fezes amolecidas. Contou que estivera trabalhando descalço nos campos de arroz alagado na semana anterior. Ao exame, apresentava-se levemente desorientado, febre de $40{ }^{\circ} \mathrm{C}$, pressão arterial $110 / 80$, pulso de $120 \mathrm{bpm}$, hiperemia conjuntival ++. Apresentava rigidez de nuca leve e hipersensibilidade muscular generalizada. Foram os únicos achados semiológicos. O laboratório mostrou elevaçóes discretas do leucograma (leucocitose polimorfonuclear) e da ureia, assim como também discretas elevaçôes da bilirrubina e da TGO. CPK moderadamente elevada e RX do tórax que evidenciou infiltrados pulmonares difusos. O EAS mostrou albuminúria +. Disso resultou o diagnóstico clínico de uma sepse por leptospirose.

Oito horas após a admissão, o paciente evoluiu com quadro de hiperpirexia grave, taquicardia, oligúria e fibrilação atrial. Foi tratado com penicilina cristalina 2.000.000UI a cada quatro horas. Depois de quarenta e oito horas, apresentou oligúria

*** significa que é uma mulher que gestou quatro vezes (G4) e pariu três vezes (P3). 
grave e instalou-se um quadro de insuficiência renal aguda e necessitou de cinco sessóes de hemodiálise. A essa altura, o anticorpo antileptospira já estava positivado.

No momento, ele está em plena recuperação, sem sequelas e seu diagnóstico final foi o de sepse causada por leptospirose e complicada com cardite, alveolite e insuficiência renal aguda.

Comentário: evitar quadros como esse exige a conscientização da comunidade sobre medidas preventivas de saúde.

\section{Estados Unidos}

CS é uma senhora de 48 anos que foi acordada por um desconforto torácico que durou cerca de uma hora, acompanhado de náuseas e sudorese. Ela permaneceu com fadiga e um pouco de falta de ar. Pensou em ir até um pronto-socorro, mas lembrou-se de que a despesa hospitalar poderia ser muito cara e que ela esperaria muito tempo pelo atendimento, uma vez que não possuía seguro-saúde. Por isso, ela não chamou nenhuma ambulância e se automedicou com um cigarro, um antiácido e repouso até que os filhos chegassem da escola. Ela morreu de infarto agudo do miocárdio antes disso acontecer.

CS é uma senhora de 48 anos que foi acordada por um desconforto torácico que durou cerca de uma hora, acompanhado por náuseas e sudorese. Ela permaneceu com fadiga e um pouco de falta de ar. Discou 192 e uma ambulância a conduziu ao hospital rural onde um ECG e as provas sanguíneas confirmaram que ela tinha sofrido um infarto agudo do miocárdio. O protocolo de suporte cardíaco avançado foi seguido e ela foi conduzida por resgate aéreo para o centro de referência coronariano mais próximo onde foi submetida imediatamente a uma revascularização e teve alta caminhando, após três dias.

Comentário: Os EUA possuem os mais sofisticados recursos de saúde disponíveis, o que não impede que quarenta milhôes de americanos estejam excluídos do acesso a eles.

\section{África do Sul}

Uma criança com malformação congênita (cloaca anal) foi encaminhada aos cirurgiōes pediátricos de um centro de cuidados terciários a cinco horas de distância do hospital rural. A consulta foi respondida com a informação de que a cirurgia somente seria possível a partir dos seis meses de idade e que deveria ser feito, como tratamento até lá a dilatação do orifício anal, duas vezes ao dia com uma vela de Helgar no 8. Esta criança precisava ser levada até uma clínica de periferia, distante duas horas de caminhada, que era atendida por um médico uma vez por semana e que náo tinha disponível a vela de Helgar. O que fazer? Após muita discussão com a mãe, o compromisso assumido foi de que ela traria a criança à clínica a cada duas SEMANAS e que o médico, estaria assegurado, traria o instrumento para realizar o procedimento. A criança terminou sendo encaminhada na época certa ao centro de referência e sua cirurgia foi malsucedida. Que dizer da qualidade desse cuidado?

\section{Grupo de Trabalho da Wonca sobre Medicina Rural}

A WONCA é um grupo de trabalho composto por até 20 membros com, pelo menos, dois representantes de cada uma das regiōes do mundo: Europa, Ásia, África, América do Norte, América do Sul, Australásia/Pacífico. Estes membros são identificados através de associaçôes membro WONCA em cada região, com uma exigência de que cada membro seja um profissional rural ou tenha uma estreita associação com a prática rural. O Grupo de Trabalho está empenhado em alcançar a equidade de gênero.

O presidente do Grupo de Trabalho é eleito pelos membros do Grupo de Trabalho para um mandato de três anos, com início no ano de cada Conferência Mundial WONCA.

Visão

Saúde para todas as pessoas ao redor do mundo rural

Missão

Melhorar a saúde rural em todo o mundo 


\section{Objetivos}

1. Facilitar a comunicação e formação de redes de medicina geral rural em todo o mundo, tanto individualmente como através de organizaçóes médicos de família rurais e grupos de interesse;

2. Representar os médicos de família rural dentro da WONCA, Conselho da WONCA, Comissóes Permanentes, Grupos de Trabalho e Organizaçóes Membros;

3. Através da WONCA, sobre questões de saúde rural, contactar a Organização Mundial de Saúde e outros órgãos internacionais relevantes;

4. Colaborar com as organizaçóes de médicos rurais;

5. Tratar de questões de importância para os médicos de família rurais, incluindo desenvolver:

5.1. Sistemas de saúde rurais cuidados com um financiamento adequado para atender às necessidades da comunidade; 5.2. Integração do médico de família na oferta de cuidados de saúde com foco na atenção primária no meio rural; 5.3. Participação da comunidade, incluindo uma abordagem multissetorial aos cuidados de saúde e promoção da saúde nas comunidades rurais;

5.4. Estratégias para melhorar o estado e a saúde das mulheres rurais em todo o mundo;

5.5. Modelos de força de trabalho rural de saúde;

5.6. Recrutamento, retenção e apoiar estratégias para os profissionais rurais;

5.7. Educação e formação para a prática médica rural; e

5.8. Pesquisa em saúde rural e prática rural, incluindo a criação de parcerias de pesquisa e desenvolvimento envolvendo indivíduos e organizaçôes em diferentes países.

Tradução para a versão brasileira: Dijon Hosana Silva e Leonardo Cançado Monteiro Savassi

Rio de Janeiro: SBMFC, 2011 